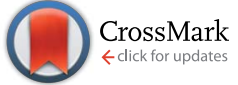

Cite this: RSC Adv., 2016, 6, 55343
Received 13th April 2016

Accepted 2nd June 2016

DOI: 10.1039/c6ra09519d

www.rsc.org/advances

\section{Mechanical and physical properties of poly(vinyl alcohol) microfibers fabricated by a microfluidic approach}

\begin{abstract}
Farrokh Sharifi, ${ }^{a}$ Zhenhua Bai, ${ }^{a}$ Reza Montazami ${ }^{\text {ab }}$ and Nastaran Hashemi ${ }^{\star a b c}$
A microfluidic platform was used to fabricate continuous and non-rounded polyvinyl alcohol (PVA) microfibers. We showed that the size and cross-section of the PVA fibers can be controlled by changing the PVA concentration in dimethyl sulfoxide (DMSO) and flow rate ratio between the core and sheath fluids. The PVA concentration was varied from $6 \%$ to $12 \%$, and the sheath-to-core flow rate ratio used for this study was in the range of $500: 5$ to $500: 20$. The aspect ratio of the fibers became larger when the PVA concentration increased and the flow rate ratio decreased. Additionally, we simulated the microfluidic fiber fabrication process and the results were consistent with the experimental results. The dissolution of the PVA fibers fabricated with different characteristics was also studied. It was shown that increasing the PVA concentration and decreasing the flow rate ratio increased the dissolution time of the fibers in DI water. A tensile test was conducted to obtain the stress-strain curves for different types of fibers. The results showed that a wide range of mechanical properties can be achieved by changing the PVA concentration and the flow rate ratio. The increase of PVA concentration from $6 \%$ to $12 \%$ enhanced the tensile stress at break and Young's modulus by a factor of 4.9 and 2.02, respectively. The mechanical strength of the fibers was shown to drop when the flow rate ratio decreased.
\end{abstract}

\section{Introduction}

Nowadays, polymeric fibers are attracting more and more attention due to their remarkable characteristics such as extremely large surface area to volume ratio, flexibility in surface functionalities, and superior mechanical performance (e.g., stiffness and tensile strength) compared with any other known form of the material. ${ }^{\mathbf{1 - 4}}$ These outstanding properties make the polymer fibers optimal candidates for many important applications such as tissue engineering, cell encapsulation, wound dressings, and drug release. ${ }^{5-9}$

Several different fiber fabrication approaches exist such as microfluidic, electrospinning, and wet spinning. ${ }^{10,11}$ Microfluidics is an emerging approach that uses small amount of samples for a wide range of applications from biomedical systems to energy devices. ${ }^{\mathbf{1 2 - 1 7}}$ The microfluidic method is a simple, rapid, and low-cost method for producing fibers, which depends on a number of experimental parameters. Additionally, this approach does not need high temperature, voltage, or pressure, which can damage the living systems in the biomedical applications. ${ }^{18-21}$ The shape of the resulting fiber is

${ }^{a}$ Department of Mechanical Engineering, Iowa State University, Ames, IA 50011, USA. E-mail: nastaran@iastate.edu

${ }^{b}$ Center of Advanced Host Defense Immunobiotics and Translational Medicine, Iowa State University, Ames, IA 50011, USA

${ }^{c}$ Center of Multiphase Flow Research, Iowa State University, Ames, IA 50011, USA a function of the flow rates and the types and numbers of shaping elements in the channel walls such as various chevron grooves. $^{22-24}$

Presently, great efforts have been devoted to expand the variety of materials that can be applied to fabricate various types of structures using microfluidics. ${ }^{13,25,26}$ For instance, Thangawng et al. produced round PMMA fibers with diameters down to $300 \mathrm{~nm}$ by varying the ratio between the sheath and core flow rates using a 5-diagonal groove device. Ribbon-shaped fibers with submicron thickness were also fabricated using a 7-chevron/5-diagonal groove combination device. ${ }^{27}$ Recently, our group successfully fabricated uniform polycaprolactone (PCL) and gelatin microfibers via phase inversion solidification process. ${ }^{19,22}$ We showed that various morphologies and crosssections (round, square and ribbon) can be obtained by varying the PCL and gelatin concentrations in the core solution and the sheath-to-core flow rate ratio. It is expected that this strategy can be applied to fabricate various fibers.

Poly(vinyl alcohol) (PVA) is a nontoxic, biocompatible, hydrophilic synthetic polymer with good chemical, thermal, and mechanical stability. It also possesses a wide range of crystallinity. Recently, it was found that PVA can be used for transient electronics and bioelectronics. ${ }^{28,29}$

Previously, PVA microfibers were fabricated using an electrospinning approach. ${ }^{30-32}$ However, using electrospinning method to fabricate fibers has some disadvantages. The electrospun fibers are fabricated with only round cross-sections due to 
the surface tension between the polymer and air during formation. It is also hard to accurately align the electro-spun fibers. The fiber characteristics, such as diameter and surface properties, do not vary widely. ${ }^{33-36}$ The current PVA fibers, on the other hand, are not necessarily rounded. They can be fabricated with different aspect ratios by simply changing different parameters such as the flow rate ratio between two fluids. We showed that the non-rounded fibers results in variations in mechanical properties and increasing the surface area-tovolume ratio of the fibers which is important in applications such as tissue engineering and biosensors. ${ }^{37-39}$ Moreover, the microfluidic platform can be considered as an alternative approach to precisely control the fiber alignment. In fact, this alignment is not limited to the position of one fiber with respect to others. The microfluidic approach makes it feasible to align the polymer chain of each fiber due to the shear force exerted on the pre-polymer solution from the sheath fluid in the flow direction. The aligned fibrous scaffold can significantly guide the growth direction of the cells, which is useful in regenerating nerve tissues, blood vessels, tendons, and muscle tissue. ${ }^{\mathbf{4 0 - 4 3}} \mathrm{We}$ demonstrated that the size and surface properties of microfluidic-spun PVA fibers can be easily tuned by simply changing the 3D hydrodynamic focusing force exerted on the core fluid. In addition, the current PVA fibers made in this study can be used to encapsulate cells for some biomedical applications such as cell delivery, whereas high voltage used in electrospinning method could be harmful for the cells. Despite its advantages, the production rate in microfluidic approach is slower than that of the electrospinning. This is a result of advances in the electrospinning technique brought about during several decades of industrial use. Microfluidic approach, on the other hand, is a new method that has shown promising potential for various biomedical applications, but it may take more time for this method to be fully understood and more well-known.

PVA has been used in microfluidic fiber fabrication process before by Jeong et al. ${ }^{\mathbf{4 4}}$ However, in that study, fiber was made of 4-hydroxybutyl acrylate (4HBA), and PVA solution (PVA in DI water) was applied as a non-polymerizable fluid (sheath fluid) to adjust the shape of the fiber. In the present work, we fabricated PVA fibers using a microfluidic approach for the first time. We showed that the size, shape, dissolution, and mechanical properties of PVA microfluidic-spun fibers can be tuned by changing the PVA concentration and flow rate between the core and sheath fluids. In addition, the microfluidic fiber fabrication process was simulated using COMSOL Multiphysics to study the effect of flow rate ratio on the shape of the resulting fiber.

\section{Results and discussion}

\subsection{Microfluidic fiber fabrication}

The fabrication method for PVA microfibers utilizing a microfluidic device is shown in Fig. 1(a). Both of the fluids, i.e. the core and sheath fluids, are introduced into the channel using silicone tubing. The channel has three inlets, one for the core fluid at the center and two for the sheath fluid.

As shown in Fig. 1(b), we employed the phase inversion process to solidify the poly(vinyl alcohol) (PVA). In this process,
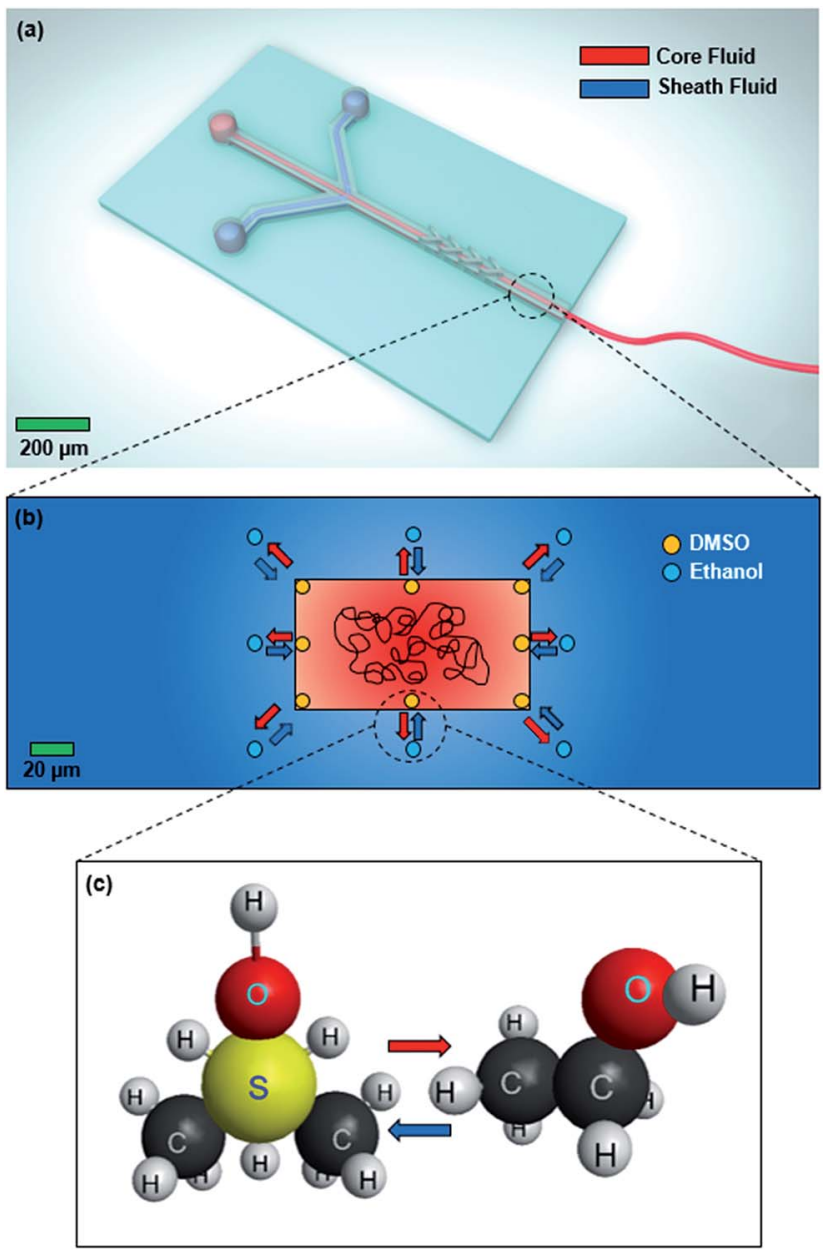

Fig. 1 (a) Schematic of the experimental setup used for microfluidic fiber fabrication. (b) Phase inversion solidification strategy applied to fabricate the microfluidic-spun PVA fibers. (c) Replacement of DMSO molecules with ethanol at the core/sheath interface, which results in aggregation and precipitation of PVA as a fiber.

dimethyl sulfoxide (DMSO), the solvent of PVA, is replaced by the sheath fluid, i.e. ethanol, at the interface between the core and sheath fluids (Fig. 1(c)). The PVA solidifies because the ethanol is miscible with DMSO and it does not dissolve the PVA. In the microfluidic channel, the lateral hydrodynamic focusing force from the sheath fluid compresses the core into a thin vertical strip, which results in increased height of the core fluid in the channel. At the same time, because of the laminar flow regime, diffusion takes place at the core/sheath fluid interface and the PVA stream rapidly solidifies as a result of the phase inversion process. Additionally, the channel consists of four chevron grooves at the downstream of the channel. Once the two fluids reach this area, the sheath fluid fills the chevron grooves due to the fact that the hydrodynamic resistance is inversely proportional to the flow rate. As a result, the sheath fluid compresses the core fluid perpendicular to the main flow direction. ${ }^{45,46}$ These vertical and lateral forces on the core fluid originate from the shear force between the core and sheath fluids due to the velocity difference at the fluid/fluid interface, which results in aligning the polymer chain in the direction of the flow. 


\subsection{Effect of PVA concentration}

One of the important parameters which plays a significant role on the resulting fiber is the concentration of PVA in the core fluid. Fig. 2 shows the viscosity of the core solution as a function of PVA concentration. Based on this figure, when the PVA concentration increases from $6 \%$ to $12 \%$, the viscosity of the core fluid rises from 78 to $297 \mathrm{cP}$. This shows the feasibility of microfluidic fiber fabrication in a wide range of viscosities.

The scanning electron microscopic (SEM) images of the fibers fabricated using different PVA concentrations are shown in Fig. 3. For this part, the flow rates of the core and sheath fluids were kept constant at $500 \mu \mathrm{L} \min ^{-1}$ and $5 \mu \mathrm{L} \mathrm{min}{ }^{-1}$, respectively. Fig. 3(a $\left.a_{1}\right)-\left(c_{1}\right)$ demonstrates that the average diameter of the fibers increases with the increase of the PVA concentration in core fluid. That is because a fixed amount of core fluid will be in the microchannel in a steady state condition when the core flow rate is constant. Thus, a more concentrated core fluid results in a larger fiber being fabricated during the phase inversion process in the channel. However, due to the fact that the microchannel has a rectangular cross-section and the shape of the fiber varies based on the flow rate ratio between the core and sheath fluids, the resulting fibers will have different cross-sections. Therefore, it is more accurate to study the size and shape of the fibers using the cross-sectional images (Fig. $\left.3\left(a_{2}\right)-\left(c_{2}\right)\right)$. The results show that the ribbon shape PVA fibers can be fabricated through the hydrodynamic focusing process. The width and height of the PVA fibers made by $6 \%$, $8 \%$, and $10 \%$ PVA are summarized in Fig. 3(d). These figures clearly illustrate the effects of both lateral and vertical forces exerted on the core fluid from the sheath fluid. Regarding the lateral force, when higher concentration of PVA is used, the core fluid covers more space of the channel due to the higher amount of PVA in DMSO. This leads to development of the fiber in a lateral direction from $13.9 \mu \mathrm{m}$ to $26.8 \mu \mathrm{m}$. On the other

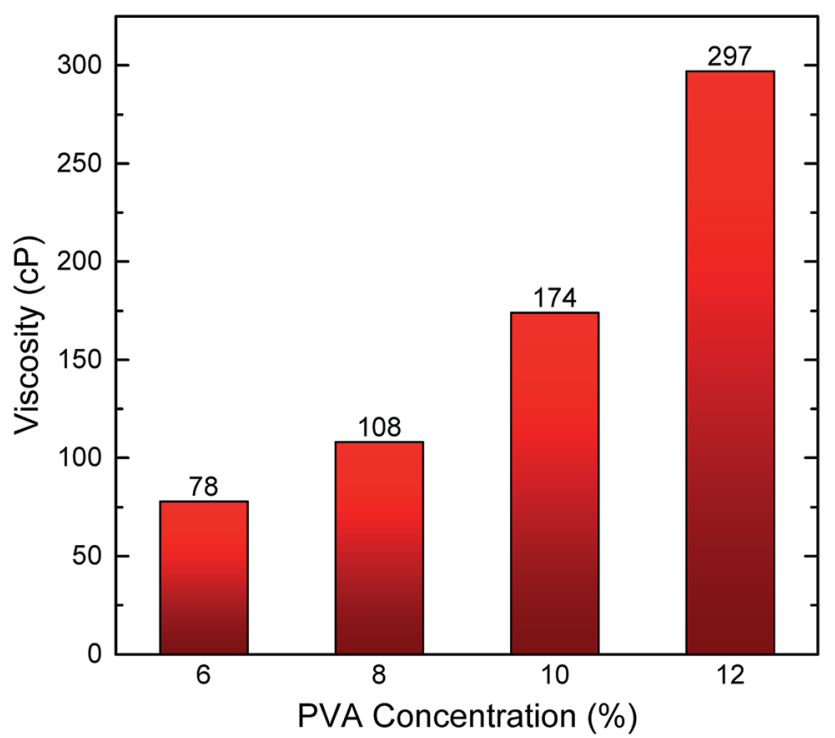

Fig. 2 Viscosity of core solution prepared by various PVA concentrations in DMSO hand, the magnitude of the vertical force is mostly dependent on the shape and height of the chevrons, which have not been changed during this study. As a result, the height of the PVA microfibers does not change significantly. When the PVA concentration increases from $6 \%$ to $8 \%$, the width of the fiber rises, whereas its height decreases such that the aspect ratio increases by a factor of 1.84 . This reveals the importance of the PVA concentration in the shape and size of the fiber.

\subsection{Effect of flow-rate ratio between the sheath and core fluids}

Using different flow rate ratios for the core and sheath fluids results in the changes of the shear force at the fluid/fluid interface. Therefore, the flow rate ratio between the core and sheath fluids plays a pivotal role in the shape and morphology of the resulting microfibers. In this section, we used the flow rate ratios in the range of $25-100$. We could not use lower flow rate ratios because the resulting shear force from the sheath fluid would not be enough to hydrodynamically focus the core fluid in the vertical and lateral directions, which would result in clogging of the channel. Fig. 4 shows the SEM images of the fibers fabricated using the sheath-to-core flow rates of $500: 5,500: 10$, and $500: 20$, respectively. In the previous section, it was shown that by using the sheath-to-core flow rate of $500: 5$ and PVA concentration in the range of $6-10 \%$, a continuous and uniform fiber can be fabricated. However, it was observed that when the PVA concentration increases to $12 \%$ and the sheath-to-core flow rate ratio is kept at $500: 5 \mu \mathrm{L} \min ^{-1}$, the large viscosity mismatch between the core and sheath fluid leads to the fabrication of a non-uniform fiber (Fig. $4\left(a_{1}\right)$ and $\left(a_{2}\right)$ ). However, Fig. $4\left(a_{1}\right)-\left(c_{1}\right)$ demonstrates that the non-uniformity of the fibers at the PVA concentration of $12 \%$ can be improved by decreasing the sheath-to-core flow rate ratio from 500:5 to 500:10 and $500: 20$. The reason for this behavior is that when the PVA concentration increases to $12 \%$, the viscosity contrast between the core and sheath fluids becomes larger. Therefore, the shear force between the core and sheath fluid exceeds the stable flow regime, which is the reason for the two viscous fluids to experience instability. To avoid this issue, we reduced the flow rate ratio between the two fluids in order to decrease the shear force at the core/sheath interface. This reveals that the shape and morphology of the fibers are dependent on the flow rate ratio between the core and sheath fluids as well as the PVA concentration in the core solution. Furthermore, the cross-sectional SEM images of the fibers are shown in Fig. $4\left(a_{2}\right)-\left(c_{2}\right)$. The width and height of the PVA fibers fabricated by the PVA concentration of $12 \%$ and different sheath-to-core flow rates of $500: 5,500: 10$, and $500: 20$ are provided in Fig. 4(d). The results demonstrate that the average width and height of the fiber at the sheath-to-core flow rate of $500: 5$ are $14.6 \mu \mathrm{m}$ and 8.6 $\mu \mathrm{m}$, respectively. The decrease of the flow rate ratio weakens the shear force of the sheath fluid on the core fluid because the relative velocity at the fluid/fluid interface reduces. Therefore, the core fluid develops in the microchannel such that the width of the resulting fiber increases by $45 \%$ at the flow rate ratio of $500: 20$, while the width of the channel negligibly varies. 

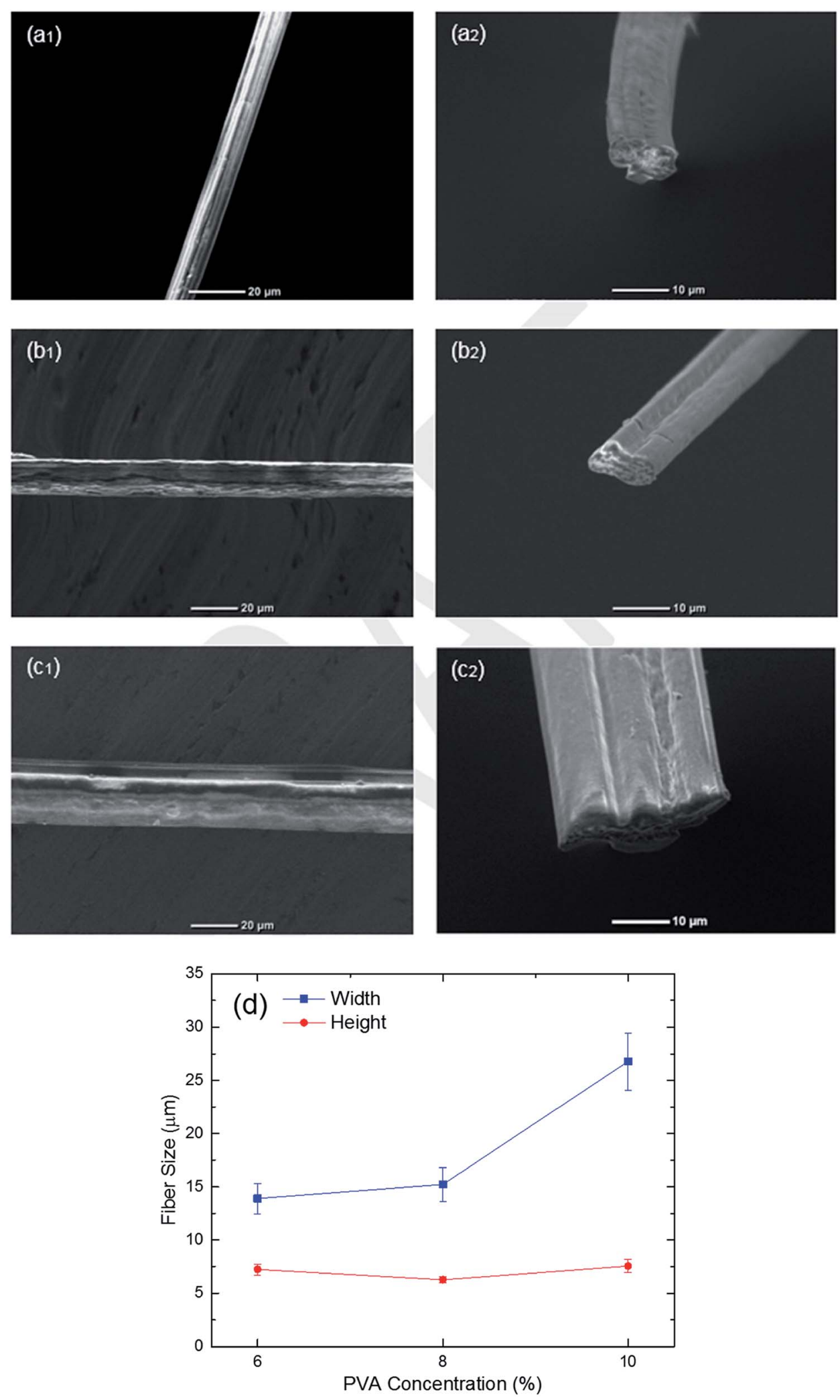

Fig. 3 Longitudinal $\left(a_{1}-c_{1}\right)$ and cross-sectional $\left(a_{2}-c_{2}\right)$ SEM images of PVA microfibers fabricated by PVA concentrations of (a) 6\%, (b) $8 \%$, and (c) $10 \%$ in DMSO solution. (d) Width and height of the fibers made by different PVA concentrations (\%). The sheath and core flow rates were set as $500 \mu \mathrm{L} \min ^{-1}$ and $5 \mu \mathrm{L} \min ^{-1}$, respectively. 

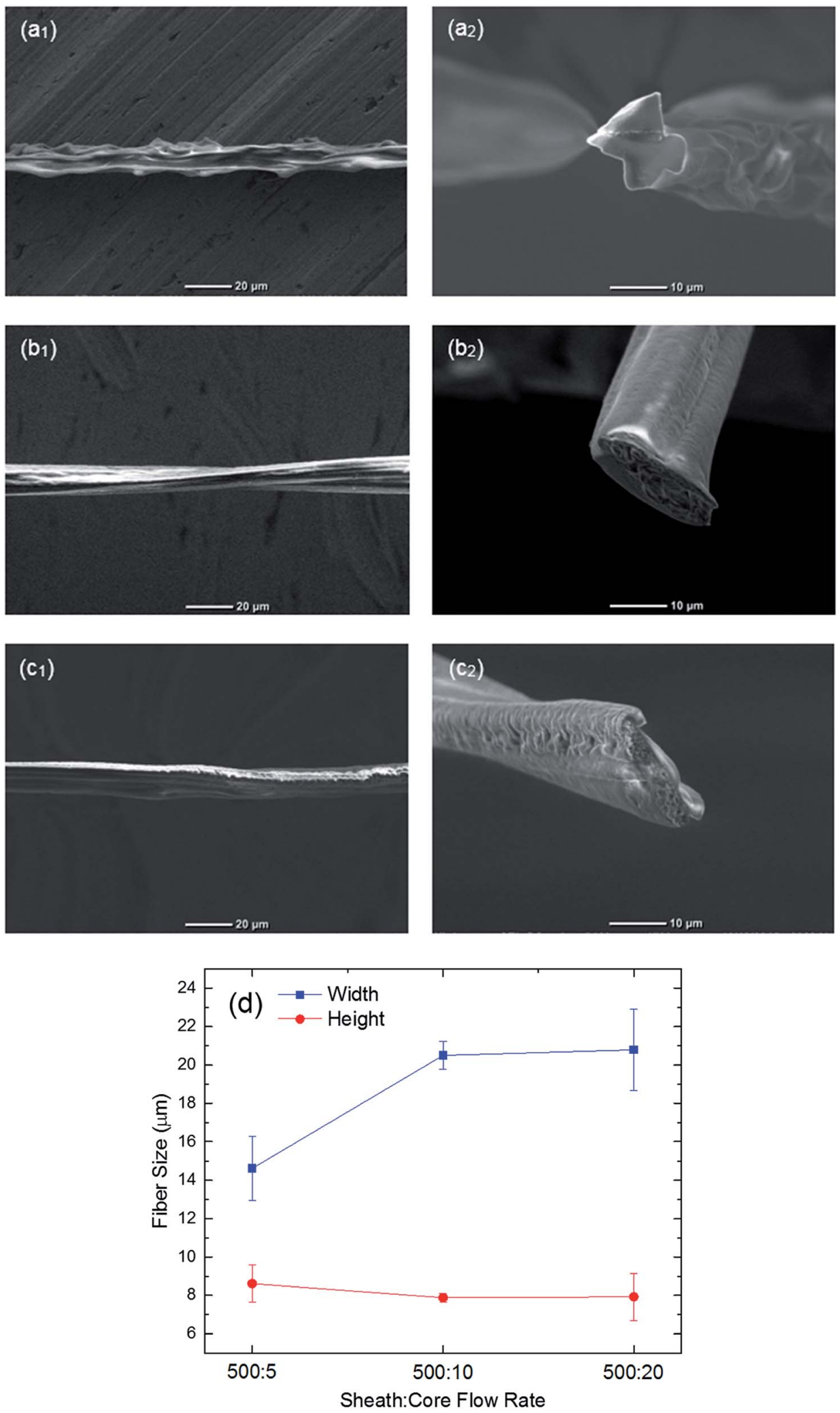

Fig. 4 Longitudinal $\left(a_{1}-c_{1}\right)$ and cross-sectional $\left(a_{2}-c_{2}\right)$ SEM images of PVA microfibers fabricated by the PVA concentration of $12 \%$ in DMSO and the sheath-to-core flow-rate ratios of (a) $500: 5$, (b) $500: 10$ and (c) $500: 20$. (d) Width and height of the fibers made by different flow rate ratios and $12 \%$ PVA concentration. 
Apart from the experimental work, the microfluidic fiber fabrication process was simulated using COMSOL multiphysics. For the simulation, one fourth of the microchannel was modeled due to the symmetric geometry of the channel and boundary conditions. The Navier Stokes and Fick equations were applied to find the velocity and concentration, respectively. Fig. 5 demonstrates the concentration distribution along the channel and at the outlet. The white and black colors represent the core and sheath fluids, respectively. Fig. 5(a) clearly illustrates both of the lateral and vertical hydrodynamic focusing forces exerted by the sheath fluid on the core fluid. At the beginning of the channel, the velocity of the sheath fluid is considerably higher than that of the core fluid, and this velocity difference intensifies in the nozzle area. As a result, the core fluid is laterally focused, and it is changed to a vertical strip. After the nozzle region, the sequential chevron grooves play a significant role in creating the vertical force causing the shear force to wrap around the core fluid, such that the core fluid is placed at the center of the channel. In this area, the hydrodynamic resistance perpendicular to the flow direction decreases and the sheath fluid fills this area as the resistance is inversely proportional to the flow rate. Thus, the core fluid is vertically focused from top and bottom, which results in the fabrication of the ribbon-shaped fibers. Based on these explanations, it is obvious that the values of the core and sheath flow rates directly affect the size and aspect ratio of the resulting fibers. For example, Fig. 5(b) and (c) shows the cross-section of the fibers at the outlet of the channel when the sheath-to-core flow rates are $500: 10$ and $500: 20$, respectively. As expected, the size and aspect ratio of the fiber increase when the flow rate ratio decreases. That is because the increase of the core flow rate reduces the relative velocity at the interface between the two fluids, which results in the decrease of the lateral shear force on the core fluid. However, the vertical force on the core fluid mostly depends on the height and number of the chevrons, which were kept constant in this study. ${ }^{46}$ Therefore, the width of the fiber enlarges and the height of the fiber is not directly affected by changing the flow rate ratio, which results in fabrication of fibers with higher aspect ratios. This behavior was observed in experimental results (Fig. $4\left(a_{2}\right)-\left(c_{2}\right)$ ), which shows a good agreement between the experimental and numerical results.

\subsection{Characterization of the PVA fibers}

The dissolution of the fibers with different characteristics was measured using UV-Vis spectroscopy and the results are shown in Fig. 6. This method was selected because the weight of the fibers is very low and the dissolution of PVA is relatively fast. Therefore, the conventional methods, such as weighing the sample at different times, are not accurate for measuring the dissolution of the fibers. However, because PVA does not have any UV-Vis absorption band, we dyed the fibers with a blue color, which gives a peak at the wavelength of $630 \mathrm{~nm}$. As an example, the result of UV-Vis spectroscopy for the dissolution of the fiber fabricated using 12\% PVA with the flow rate ratio of $500: 5$ is illustrated in Fig. 6(a). The results show that the peak at the wavelength of $630 \mathrm{~nm}$ intensifies with time, which represents the dissolving of the PVA fiber in DI water. As expected, this figure demonstrates that the dissolution of the PVA fibers is fast. The speed of dissolution can be attributed to the fact that the hydroxyl groups in PVA create hydrogen-bonding with the molecules of highly polar solvents, such as water. This work was conducted for fibers fabricated with various PVA concentrations and flow rate ratios, and the results are provided in Fig. 6(b). Based on this figure, the increase of the PVA concentration increases the dissolution time of the PVA fibers. That is expected because when the core flow rate is set at $5 \mu \mathrm{L}$ $\min ^{-1}$, the volume of the core fluid that occupies part of the channel is constant. Therefore, a more concentrated core solution gives a higher amount of PVA in the channel, which results in fabrication of larger fibers that take a longer time to be dissolved in water. Additionally, the decrease of the flow rate
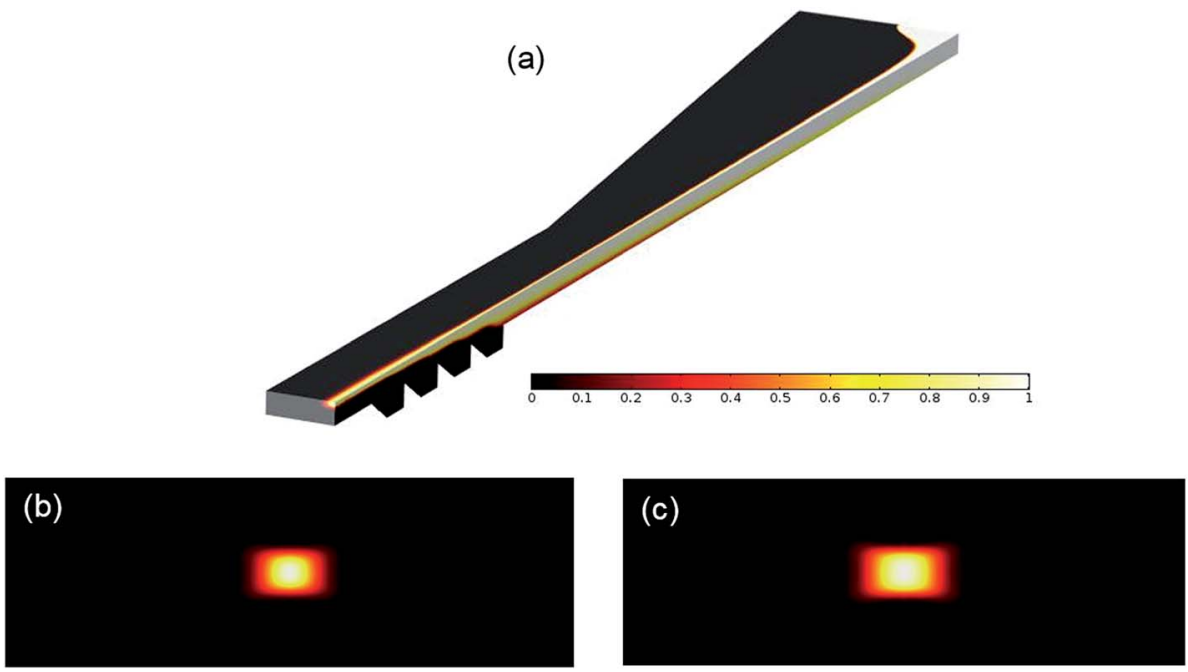

Fig. 5 The concentration ( $\mathrm{mol} \mathrm{m} \mathrm{m}^{-3}$ ) profile of the sheath and core fluids (a) along the channel and (b and c) at the outlet with the sheath-to-core flow rates of $500: 10$ and $500: 20$, respectively. The white color represents the core fluid and the sheath fluid is black in this study. 

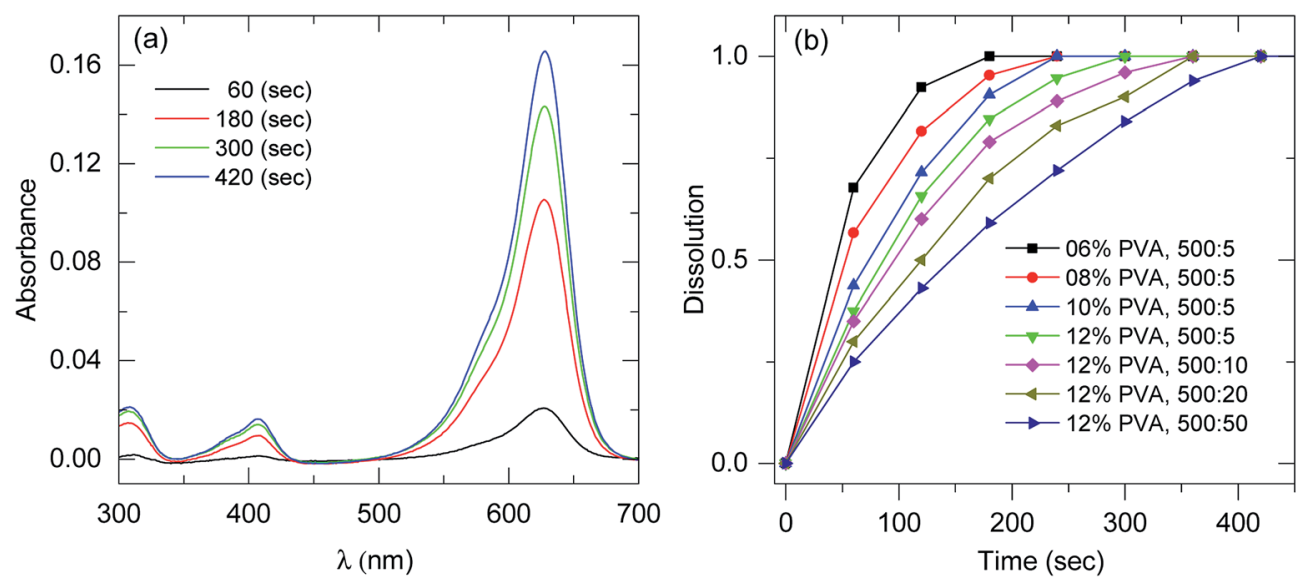

Fig. 6 Dissolution of the PVA fibers in DI water. (a) UV-Vis absorption spectra of dyed fiber with the PVA concentration of $12 \%$ in water at different times. (b) Dissolution of PVA microfibers fabricated by various PVA concentrations and flow-rates in water.

ratio between the core and sheath fluids reduces the hydrodynamic focusing force, and the average size of the fiber becomes larger. This causes the PVA fiber to last longer in water.

The mechanical properties of biomaterials are important aspects in different biomedical applications such as tissue engineering. One of the goals of using scaffolds is to be able to mimic the mechanical properties of the tissue or organ. Fig. 7 shows the results of conducting a tensile test for the fibers created with different PVA concentrations and flow rate ratios. Additionally, the values of the tensile stress at break (MPa), tensile strain at break (\%), and Young's modulus ( $\mathrm{MPa}$ ) are listed in Table 1. When the PVA concentration is $6 \%$ and the flow rate ratio between the sheath and core fluids is $500: 5$, the tensile stress at break, Young's modulus, and tensile strain at
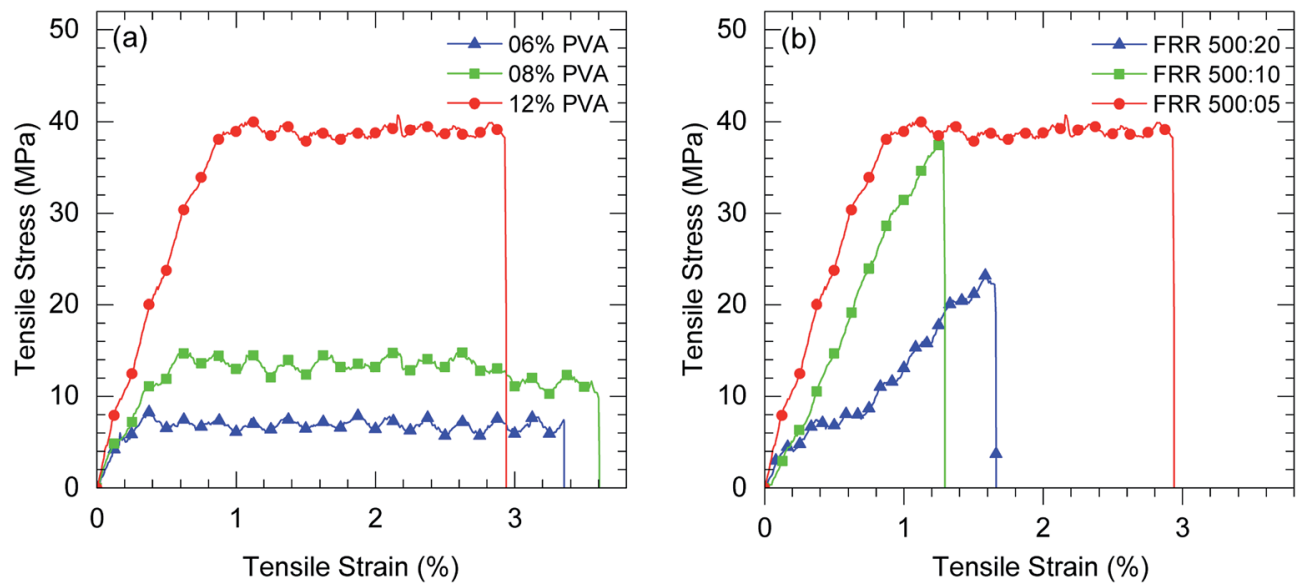

Fig. 7 Tensile properties of PVA fibers fabricated with (a) the flow rate ratio of $500: 5$ for the sheath : core fluids and various PVA concentrations in DMSO. (b) PVA concentration of $12 \%$ and different flow rate ratios.

Table 1 Mechanical properties of the fibers fabricated with various concentrations of PVA in DMSO and flow rate ratios between the core and sheath fluids

\begin{tabular}{|c|c|c|c|c|}
\hline $\begin{array}{l}\text { PVA concentration } \\
(\%)\end{array}$ & $\begin{array}{l}\text { Flow rate ratio } \\
\text { (sheath : core) }\end{array}$ & $\begin{array}{l}\text { Tensile stress } \\
\text { at break (MPa) }\end{array}$ & $\begin{array}{l}\text { Tensile strain } \\
\text { at break }(\%)\end{array}$ & $\begin{array}{l}\text { Young's } \\
\text { modulus (MPa) }\end{array}$ \\
\hline 6 & $500: 5$ & 8.30 & 3.35 & 2112.9 \\
\hline 12 & $500: 5$ & 40.71 & 2.94 & 4265.1 \\
\hline 12 & $500: 10$ & 37.81 & 1.29 & 3261.5 \\
\hline 12 & $500: 20$ & 23.15 & 1.66 & 1003.4 \\
\hline
\end{tabular}


break (\%) are 8.3 $\mathrm{MPa}, 2112.9 \mathrm{MPa}$, and 3.35, respectively. By increasing the PVA concentration to $12 \%$, the tensile stress at break and Young's modulus are significantly enhanced by $390 \%$ and $102 \%$, respectively. Similarly, the tensile strain at break (\%) improves by increasing the PVA concentration to $10 \%$. However, it reduces with further increase in the PVA concentration. All of the measurements were done at room temperature (below the glass transition temperature of the PVA at $85^{\circ} \mathrm{C}$ ), which results in low tensile strain at break for all types of the PVA fibers. In addition, when the flow rate ratio between the core and sheath fluids decreases from $500: 5$ to $500: 20$, the tensile stress at break, tensile strain at break, and Young's modulus drop by a factor of $1.76,1.77$, and 4.25 , respectively. This can be due to the change of the cross-section of the fiber. As shown in Fig. 4, when the flow rate ratio decreases, the aspect ratio of the fiber increases and the ribbon shape fiber is fabricated. In ribbon shape fibers, one side of the cross-section of the fiber is smaller than the other one. Therefore, when the fiber is under the tensile load and the cross-section of the sample decreases, the smaller side is weaker, which leads to sample failure in lower loads and strains. This kind of behavior was observed for the thiol-ene and thiol-yne click fibers as well. ${ }^{37}$ Nevertheless, the surface area of the ribbon shape fibers is larger than that of the square fibers, which is an important aspect for fibrous scaffolds to support the growth of more cells in tissue engineering.

There are some studies in which PVA fibers were fabricated using electrospinning method..$^{3,36,47,48}$ However, there is no report about the mechanical properties of single electrospun PVA fibers. In fact, it is common to measure the mechanical properties of the fibrous mats instead in this method. After comparing the results of this study with those reported for electrospun PVA mats, it was found that the microfluidic spun PVA fibers have a higher tensile stress at break and Young's modulus, but lower tensile strain at break. The reason for higher tensile stress might be due to the fact that the shear force exerted on the prepolymer solution align the polymer chain in the flow direction. It was expected to obtain lower strain (\%) compared to the electrospun PVA mats because multiple fibers support each other and cause the mats to break at higher strain. In addition, higher strain of PVA mats can be due to the fact that the electrospun fibers are made randomly and most of them are not in the same direction as the tensile test. Consequently, they will break at higher strain.

Apart from fibrous mats, there are some reports that the mechanical properties of PVA films were measured. ${ }^{28,49-56}$ It was found that the values of tensile stress at break and Young's modulus are comparable with the ones reported in the literature for the PVA films. However, the tensile strain at break of the PVA films is larger. That might be related to the fabrication process. In this paper, the phase inversion solidification strategy was used to fabricate fiber. This strategy caused the molecules of DMSO and ethanol to be replaced which creates a porous fiber, whereas the PVA films made by different methods have lower porosity. Additionally, when a defect occurs in both PVA fiber and film, it propagates until the sample breaks. Because the average cross section area of the films is significantly larger than that of the fibers, the defect has to propagate more to completely break the sample, which results in higher strain at break (\%) for the film.

\section{Conclusions}

The PVA microfiber was fabricated using a microfluidic approach. The effects of PVA concentration in the core fluid and flow rate ratio between the core and sheath fluid were investigated. The size, cross-section, dissolution of the fiber in DI water, as well as the mechanical properties of the fibers, were studied. It was found that when a higher PVA concentration is used, the size of the fiber increases, the dissolution of the fiber in water takes more time, and the mechanical properties are improved. When the PVA concentration increases from $6 \%$ to $12 \%$, the tensile stress at break and Young's modulus are enhanced by $390 \%$ and $102 \%$, respectively. On the other hand, when the flow rate ratio between the core and sheath fluids decreases, the size and aspect ratio of the fiber become larger, the fiber lasts longer in the water, and all of the tensile properties of the fiber drop. It was observed that the tensile stress at break, tensile strain at break, and Young's modulus can be enhanced by a factor of $1.76,1.77$, and 4.25 when the flow rate ratio increases from 25 to 100 . Once again, it was proved that the microfluidic fiber fabrication is a promising platform to provide an accurate control on the size, shape, dissolution, and mechanical properties of the fibers, which could be useful in different biomedical applications.

\section{Experimental section}

\subsection{Chemicals and materials}

Poly(vinyl alcohol) (PVA) ( $M_{\mathrm{w}}: 61000 \mathrm{~g} \mathrm{~mol}^{-1}$, 98.0-98.8 mol\% hydrolysis) and absolute ethanol were purchased from SigmaAldrich. Dimethyl sulfoxide (DMSO) was purchased from Fisher Scientific. The Sylgard 184 Elastomer Base and Curing Agents were purchased from Dow Corning Corporation, Midland, MI.

\subsection{Microfluidic channel device design}

A poly(methyl methacrylate) (PDMS) channel was used in this study, which is a transparent and biocompatible elastomer. In order to make the channel, the Sylgard 184 Elastomer Base and Curing Agents (PDMS pre-polymers) in a $10: 1$ ratio were stirred and poured onto two molds made of a SU8 photoresistpatterned silicon wafer, and cured at $80{ }^{\circ} \mathrm{C}$ for $20 \mathrm{~min}$. Then the PDMS layers were peeled off and one of them was punched to create a connection between the inlet of the channel and the syringes which include the core and sheath solutions. Finally, the plasma treatment was applied to bond the two PDMS layers together. The microchannel is symmetric with one inlet for the core fluid, two inlets for the sheath fluid, and four chevron grooves. The dimensions of the channel are $130 \mu \mathrm{m} \times 390 \mu \mathrm{m}$ (height $\times$ width). The height and width of the grooves are $100 \mu \mathrm{m}$ and $65 \mu \mathrm{m}$, respectively, and their angle with respect to the main direction of the flow is 45 degrees. 


\subsection{PVA fiber fabrication}

In order to prepare the core solution, $25 \mathrm{~mL}$ of DMSO was heated to $120{ }^{\circ} \mathrm{C}$ within $20 \mathrm{~min}$, and then PVA powders were added into the DMSO under vigorous stirring. After stirring for 2 hours, the mixture was cooled to room temperature. This process was repeated to prepare the core solutions with the PVA concentration in the range of 6-12 wt\%. Absolute ethanol was applied as sheath fluid in this study. The core and sheath fluids were introduced into the channel using the $3 \mathrm{~mL}$ and $60 \mathrm{~mL}$ plastic syringes (BD Biosciences) for the core and sheath fluids, respectively, through a double syringe pump (Cole-Parmer, Veron Hillss, IL). The flow rates of the core fluid were in the range of $5-50 \mu \mathrm{L} \min ^{-1}$, whereas the constant flow rate of

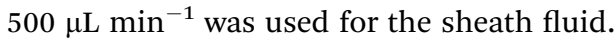

\subsection{Computational model}

The microfluidic fiber fabrication process was simulated using the COMSOL Multiphysics (COMSOL, Inc., Burlington, MA) software. The purpose of the simulation was to study the effect of various flow rate ratios between the core and sheath fluids on the fiber shape and compare the numerical and experimental results. It was assumed that the process is steady-state at room temperature, and the fluids are incompressible. The flow and diffusion modules were coupled in COMSOL in order to achieve the concentration distribution of the core and sheath fluids, and to estimate the cross-section of the resulting fibers at the outlet. First, the velocity and pressure distributions were found for the channel, and then, the results were used to obtain the concentration profile of the fluids along the channel as well as the outlet.

\subsection{Characterization}

The viscosities of core solutions with different PVA concentrations were measured using a digital viscometer (DV-E, Brookfield Engineering Laboratories, Inc., Middleboro, MA). The size, shape, and morphology of the PVA microfibers were studied using field emission scanning electron microscopy (FE-SEM) (JSM-6700F) at an acceleration voltage of $5 \mathrm{kV}$. In order to obtain the cross-sectional SEM images, the fibers were cut with a sharp razor. The dissolution of fibers were measured using UV-Vis spectrometer (PerkinElmer Lambda 25). In this part, the non-fluorescent blue dye (105002 Standard Blue Powder, Bright Dyes) with the absorbance wavelength of $630 \mathrm{~nm}$ was used in the core fluid. After calibration, the dissolution rates of the fibers with different characteristics in DI water were calculated. The tensile test was performed with an Instron Universal Testing machine (Model 5569, Instron Engineering Corp., Canton, MA) at an extension rate of $50 \mathrm{~mm} \mathrm{~min}^{-1}$ using a $2 \mathrm{kN}$ load cell. For each test, one single fiber was attached on a paper frame in order to be gripped tightly by the Instron machine. The samples were prepared such that the distance between the two clamps is $20 \mathrm{~mm}$ at the beginning. Before starting the test, the two sides of the frame were cut to obtain the stress-strain curve of the fibers without the effects of the paper frame. The Bluehill software was used to control the Instron machine and get the results of the test.

\section{Acknowledgements}

This work was funded in part by the Office of Naval Research Grant N000141612246, Iowa State University Presidential Initiative for Interdisciplinary Research, and Iowa State University Health Research Initiative (ISU-HRI). The authors would also like to thank Ashley Christopherson for illustrating the microfluidic fiber fabrication process.

\section{References}

1 X. Shi, S. Ostrovidov, Y. Zhao, X. Liang, M. Kasuya, K. Kurihara, K. Nakajima, H. Bae, H. Wu and A. Khademhosseini, Microfluidic spinning of cell-responsive grooved microfibers, Adv. Funct. Mater., 2015, 25(15), 2250-2259.

2 S.-K. Chae, E. Kang, A. Khademhosseini and S.-H. Lee, Micro/ nanometer-scale fiber with highly ordered structures by mimicking the spinning process of silkworm, Adv. Mater., 2013, 25(22), 3071-3078.

3 M. A. Daniele, K. Radom, F. S. Ligler and A. A. Adams, Microfluidic fabrication of multiaxial microvessels via hydrodynamic shaping, RSC Adv., 2014, 4(45), 23440-23446.

4 M. A. Daniele, A. A. Adams, J. Naciri, S. H. North and F. S. Ligler, Interpenetrating networks based on gelatin methacrylamide and peg formed using concurrent thiol click chemistries for hydrogel tissue engineering scaffolds, Biomaterials, 2014, 35(6), 1845-1856.

5 M. A. Daniele, D. A. Boyd, A. A. Adams and F. S. Ligler, Microfluidic strategies for design and assembly of microfibers and nanofibers with tissue engineering and regenerative medicine applications, Adv. Healthcare Mater., 2015, 4(1), 11-28.

6 Y. Jun, E. Kang, S. Chae and S. H. Lee, Microfluidic spinning of micro- and nano-scale fibers for tissue engineering, $L a b$ Chip, 2014, 14(13), 2145-2160.

7 S. Y. Ahn, C. H. Mun and S. H. Lee, Microfluidic spinning of fibrous alginate carrier having highly enhanced drug loading capability and delayed release profile, $R S C A d v$., 2015, 5(20), 15172-15181.

8 J. J. Kaufman, R. Ottman, G. Tao, S. Shabahang, E.-H. Banaei, X. Liang, S. G. Johnson, Y. Fink, R. Chakrabarti and A. F. Abouraddy, In-fiber production of polymeric particles for biosensing and encapsulation, Proc. Natl. Acad. Sci. U. S. A., 2013, 110(39), 15549-15554.

9 D. Lim, E. Lee, H. Kim, S. Park, S. Baek and J. Yoon, Multi stimuli-responsive hydrogel microfibers containing magnetite nanoparticles prepared using microcapillary devices, Soft Matter, 2015, 11(8), 1606-1613.

10 A. Tamayol, M. Akbari, N. Annabi, A. Paul, A. Khademhosseini and D. Juncker, Fiber-based tissue engineering: progress, challenges, and opportunities, Biotechnol. Adv., 2013, 31(5), 669-687.

11 M. Akbari, A. Tamayol, V. Laforte, N. Annabi, A. H. Najafabadi, A. Khademhosseini and D. Juncker, Composite living fibers for creating tissue constructs using textile techniques, Adv. Funct. Mater., 2014, 24(26), 40604067. 
12 G. M. Whitesides, The origins and the future of microfluidics, Nature, 2006, 442(7101), 368-373.

13 J. D. Caplin, N. G. Granados, M. R. James, R. Montazami and N. Hashemi, Microfluidic organ-on-a-chip technology for advancement of drug development and toxicology, Adv. Healthcare Mater., 2015, 4(10), 1426-1450.

14 F. Sharifi, S. Ghobadian, F. R. Cavalcanti and N. Hashemi, Paper-based devices for energy applications, Renewable Sustainable Energy Rev., 2015, 52, 1453-1472.

15 P. Asrar, M. Sucur and N. Hashemi, Multi-pixel photon counters for optofluidic characterization of particles and microalgae, Biosensors, 2015, 5(2), 308.

16 C. Meis, R. Montazami and N. Hashemi, Ionic electroactive polymer actuators as active microfluidic mixers, Anal. Methods, 2015, 7(24), 10217-10223.

17 N. Hashemi, J. M. Lackore, F. Sharifi, P. J. Goodrich, M. L. Winchell and N. Hashemi, A paper-based microbial fuel cell operating under continuous flow condition, Technology, 2016, DOI: 10.1142/S2339547816400124.

18 P. J. Goodrich, F. Sharifi and N. Hashemi, Rapid prototyping of microchannels with surface patterns for fabrication of polymer fibers, RSC Adv., 2015, 5(87), 71203-71209.

19 F. Sharifi, D. Kurteshi and N. Hashemi, Microfluidic fabrication of highly structured polycaprolactone (pcl) fibers, J. Mech. Behav. Biomed. Mater., 2016, 61, 530-540.

20 E. Kang, G. S. Jeong, Y. Y. Choi, K. H. Lee, A. Khademhosseini and S. H. Lee, Digitally tunable physicochemical coding of material composition and topography in continuous microfibres, Nat. Mater., 2011, 10(11), 877-883.

21 M. A. Daniele, S. H. North, J. Naciri, P. B. Howell, S. H. Foulger, F. S. Ligler and A. A. Adams, Rapid and continuous hydrodynamically controlled fabrication of biohybrid microfibers, Adv. Funct. Mater., 2013, 23(6), 698704.

22 Z. H. Bai, J. M. M. Reyes, R. Montazami and N. Hashemi, Onchip development of hydrogel microfibers from round to square/ribbon shape, J. Mater. Chem. A, 2014, 2(14), 48784884.

23 D. A. Boyd, A. R. Shields, P. B. Howell and F. S. Ligler, Design and fabrication of uniquely shaped thiol-ene microfibers using a two-stage hydrodynamic focusing design, Lab Chip, 2013, 13(15), 3105-3110.

24 M. Hu, R. Deng, K. M. Schumacher, M. Kurisawa, H. Ye, K. Purnamawati and J. Y. Ying, Hydrodynamic spinning of hydrogel fibers, Biomaterials, 2010, 31(5), 863-869.

25 S. Utech, R. Prodanovic, A. S. Mao, R. Ostafe, D. J. Mooney and D. A. Weitz, Microfluidic generation of monodisperse, structurally homogeneous alginate microgels for cell encapsulation and 3d cell culture, Adv. Healthcare Mater., 2015, 4(11), 1628-1633.

26 L. Mazutis, R. Vasiliauskas and D. A. Weitz, Microfluidic production of alginate hydrogel particles for antibody encapsulation and release, Macromol. Biosci., 2015, 15(12), 1641-1646.

27 A. R. Shields, C. M. Spillmann, J. Naciri, P. B. Howell, A. L. Thangawng and F. S. Ligler, Hydrodynamically directed multiscale assembly of shaped polymer fibers, Soft Matter, 2012, 8(24), 6656-6660.

28 H. Acar, S. Çınar, M. Thunga, M. R. Kessler, N. Hashemi and R. Montazami, Study of physically transient insulating materials as a potential platform for transient electronics and bioelectronics, Adv. Funct. Mater., 2014, 24(26), 41354143.

29 R. Jamshidi, S. Çinar, Y. Chen, N. Hashemi and R. Montazami, Transient bioelectronics: electronic properties of silver microparticle-based circuits on polymeric substrates subjected to mechanical load, $J$. Polym. Sci., Part B: Polym. Phys., 2015, 53(22), 1603-1610.

30 F. Mano, I. M. Aroso, S. Barreiros, J. P. Borges, R. L. Reis, A. R. C. Duarte and A. Paiva, Production of poly(vinyl alcohol) (pva) fibers with encapsulated natural deep eutectic solvent (nades) using electrospinning, ACS Sustainable Chem. Eng., 2015, 3(10), 2504-2509.

31 V. M. Merkle, P. L. Tran, M. Hutchinson, K. R. Ammann, K. DeCook, X. Wu and M. J. Slepian, Core-shell pva/gelatin electrospun nanofibers promote human umbilical vein endothelial cell and smooth muscle cell proliferation and migration, Acta Biomater., 2015, 27, 77-87.

32 A. M. M. Sousa, H. K. S. Souza, J. Uknalis, S.-C. Liu, M. P. Gonçalves and L. Liu, Electrospinning of agar/pva aqueous solutions and its relation with rheological properties, Carbohydr. Polym., 2015, 115, 348-355.

33 S. Yao, Y. Li, Z. Zhou and H. Yan, Graphene oxide-assisted preparation of poly(vinyl alcohol)/carbon nanotube/ reduced graphene oxide nanofibers with high carbon content by electrospinning technology, RSC Adv., 2015, 5(111), 91878-91887.

34 Y. Zhao, Z. Fan, M. Shen and X. Shi, Capturing hepatocellular carcinoma cells using lactobionic acidfunctionalized electrospun polyvinyl alcohol/ polyethyleneimine nanofibers, $R S C A d v .$, 2015, 5(86), $70439-70447$.

35 R. Najafi-Taher, M. A. Derakhshan, R. Faridi-Majidi and A. Amani, Preparation of an ascorbic acid/pva-chitosan electrospun mat: a core/shell transdermal delivery system, RSC Adv., 2015, 5(62), 50462-50469.

36 S. Ghobadi, S. Sadighikia, M. Papila, F. C. Cebeci and S. A. Gursel, Graphene-reinforced poly(vinyl alcohol) electrospun fibers as building blocks for high performance nanocomposites, RSC Adv., 2015, 5(103), 85009-85018.

37 D. A. Boyd, A. R. Shields, J. Naciri and F. S. Ligler, Hydrodynamic shaping, polymerization, and subsequent modification of thiol click fibers, ACS Appl. Mater. Interfaces, 2013, 5(1), 114-119.

38 M. A. Daniele, D. A. Boyd, D. R. Mott and F. S. Ligler, 3d hydrodynamic focusing microfluidics for emerging sensing technologies, Biosens. Bioelectron., 2015, 67, 25-34.

39 M. A. Daniele, D. A. Boyd, A. A. Adams and F. S. Ligler, Microfluidic strategies for design and assembly of microfibers and nanofibers with tissue engineering and regenerative medicine applications, Adv. Healthcare Mater., 2015, 4(1), 11-28. 
40 F. Sharifi, B. B. Patel, A. K. Dzuilko, D. S. Sakaguchi, R. Montazami and N. Hashemi, Microfluidic spun poly( $\varepsilon^{-}$ caprolactone) (pcl) microfibrous scaffolds to navigate neural stem cells for regenerative biomedical applications, 2015.

41 E. Kang, Y. Y. Choi, S.-K. Chae, J.-H. Moon, J.-Y. Chang and S.-H. Lee, Microfluidic spinning of flat alginate fibers with grooves for cell-aligning scaffolds, Adv. Mater., 2012, 24(31), 4271-4277.

42 C. M. Hwang, A. Khademhosseini, Y. Park, K. Sun and S.-H. Lee, Microfluidic chip-based fabrication of plga microfiber scaffolds for tissue engineering, Langmuir, 2008, 24(13), 6845-6851.

43 N. Reddy and Y. Yang, Microfluidic spinning of alginate fibers, Innovative biofibers from renewable resources, Springer Berlin Heidelberg, 2015, pp. 151-154.

44 W. Jeong, J. Kim, S. Kim, S. Lee, G. Mensing and D. J. Beebe, Hydrodynamic microfabrication via "on the fly" photopolymerization of microscale fibers and tubes, $L a b$ Chip, 2004, 4(6), 576-580.

45 N. Hashemi, J. P. B. Howell, J. S. Erickson, J. P. Golden and F. S. Ligler, Dynamic reversibility of hydrodynamic focusing for recycling sheath fluid, Lab Chip, 2010, 10(15), 1952-1959.

46 P. B. Howell Jr, J. P. Golden, L. R. Hilliard, J. S. Erickson, D. R. Mott and F. S. Ligler, Two simple and rugged designs for creating microfluidic sheath flow, Lab Chip, 2008, 8(7), 1097-1103.

47 D. Yang, Y. Li and J. Nie, Preparation of gelatin/pva nanofibers and their potential application in controlled release of drugs, Carbohydr. Polym., 2007, 69(3), 538-543.

48 J. S. Jeong, J. S. Moon, S. Y. Jeon, J. H. Park, P. S. Alegaonkar and J. B. Yoo, Mechanical properties of electrospun pva/ mwnts composite nanofibers, Thin Solid Films, 2007, 515(12), 5136-5141.
49 Y. Xu, W. Hong, H. Bai, C. Li and G. Shi, Strong and ductile poly(vinyl alcohol)/graphene oxide composite films with a layered structure, Carbon, 2009, 47(15), 3538-3543.

50 A. K. Sonker, H. D. Wagner, R. Bajpai, R. Tenne and X. Sui, Effects of tungsten disulphide nanotubes and glutaric acid on the thermal and mechanical properties of polyvinyl alcohol, Compos. Sci. Technol., 2016, 127, 47-53.

51 H.-L. Ma, L. Zhang, Y. Zhang, S. Wang, C. Sun, H. Yu, X. Zeng and M. Zhai, Radiation preparation of graphene/carbon nanotubes hybrid fillers for mechanical reinforcement of poly(vinyl alcohol) films, Radiat. Phys. Chem., 2016, 118, 21-26.

52 H. Liu, P. Bandyopadhyay, N. H. Kim, B. Moon and J. H. Lee, Surface modified graphene oxide/poly(vinyl alcohol) composite for enhanced hydrogen gas barrier film, Polym. Test., 2016, 50, 49-56.

53 X. Yang, L. Li, S. Shang and X.-m. Tao, Synthesis and characterization of layer-aligned poly(vinyl alcohol)/ graphene nanocomposites, Polymer, 2010, 51(15), 34313435.

54 L. Liu, A. H. Barber, S. Nuriel and H. D. Wagner, Mechanical properties of functionalized single-walled carbon-nanotube/ poly(vinyl alcohol) nanocomposites, Adv. Funct. Mater., 2005, 15(6), 975-980.

55 B. Shen, W. Zhai, D. Lu, J. Wang and W. Zheng, Ultrasonication-assisted direct functionalization of graphene with macromolecules, RSC Adv., 2012, 2(11), 4713-4719.

56 P. May, U. Khan, A. O'Neill and J. N. Coleman, Approaching the theoretical limit for reinforcing polymers with graphene, J. Mater. Chem., 2012, 22(4), 1278-1282. 